\title{
Psychotropic medication and breast-feeding
}

\section{Dora Kohen}

\begin{abstract}
Adverse effects of psychotropic medication on breast-fed infants have not been studied in controlled and systematic research. Existing information comes from small case series and single case reports. These limited data confirm that psychotropics are excreted into breast milk and that the infant is exposed to them. In recent decades sufficient data have accumulated to allow psychiatrists to prescribe tricyclic antidepressants, selective serotonin reuptake inhibitors, conventional antipsychotics, carbamazepine and sodium valproate to breast-feeding mothers with safety. There are not sufficient data on atypical antipsychotics to allow women to breast-feed safely. Mothers on clozapine or lithium should not breast-feed. It is good practice to recommend that breast-feeding mothers requiring psychotropic medication be on a low dose of one single drug. Future research taking account of maternal mental health, psychopharmacological factors, infant physiological environment and individualised risk/benefit assessment will yield clearer responses to this complex issue.
\end{abstract}

The physical and psychological benefits of breastfeeding for the mother and the infant are numerous and range from improved and stronger bonding to better immunological protection. Breast-fed infants have fewer episodes of middle-ear and urinary tract infections and lower mortality rates than bottle-fed babies (Nulman et al, 2003). Breast milk contains nucleotides and enzymes that promote digestion and absorption of nutrients. Breast-feeding has been associated with better cognitive functioning and better performance on IQ measures at age 7-8 years (Fergusson et al, 1982; Lucas et al, 1992).

For the mother, breast-feeding causes oxytocin and prolactin release, precipitates postnatal uterine involution and suppresses ovulatory cycles.

Breast-feeding has been successfully promoted in recent decades and a significantly large group of women now believe in its benefits and enquire about the possibilities of breast-feeding even when they are on psychotropic medication. It is the duty of the prescribing psychiatrist to guide the mother, the carers, the community mental health team, the general practitioner (GP), the obstetricians and the paediatricians through the intricacies of this complex area of psychopharmacology, in which there is an increasing interest but only scant research, few case studies and limited publications.

\section{Pharmacokinetics of psychotropics in breast milk}

Researchers need information on four factors in order to understand problems related to breastfeeding by mothers taking psychotropic medication: the prescribed dose; the level of the drug in the mother's blood plasma; the level in the breast milk; and the levels in the infant's serum. The amount of drug excreted in the milk is important in evaluating any drug-induced toxicity in the infant.

The medication's diffusion across membranes, its molecular weight and its lipophilicity each play an important role in determining the amount that enters the breast milk (Nulman et al, 2003).

The milk that comes out towards the end of a feed (hind milk) has a higher concentration of lipids than the thinner foremilk. Consequently, hind milk will contain a greater concentration of any lipid-soluble drug. Colostrum, produced only during the first few days after birth, has a higher protein level and will therefore contain a greater concentration of proteinbound drugs.

To evaluate infant exposure to a drug the concentration of the medication or its metabolite are measured simultaneously in the mother's plasma and breast milk. A milk/plasma ratio greater than 1

Dora Kohen is Professor of Women's Mental Health at the Lancashire Postgraduate School of Medicine and Health (Leigh Infirmary, Leigh, Lancashire WN7 1HS, UK. E-mail: dorakohen@doctors.org.uk) and a consultant psychiatrist in perinatal psychiatry in Lancashire. Her interests are psychiatric services for women, and motherhood and severe mental illness. 
suggests a high likelihood that the infant will be exposed to the drug (Suri et al, 1998). However, it is important to note that a milk/plasma ratio calculated on one single occasion and independent of other risks may not be sufficiently significant. There are other important factors such as the drug's dosage and frequency, its pharmacodynamics and pharmacokinetics, and the timing of the dose in relation to the infant's feeding patterns. Taking several measurements at different times of the day may provide a better picture.

Infants metabolise medication transmitted via breast milk, but this varies with each individual. Preterm immature infants are more sensitive and may have immature liver function, and they should therefore not be exposed to any psychotropic medication through the breast milk.

The infants' hepatic, cardiac and renal functions should be checked before they are breast-fed by mothers treated with psychotropics.

The infant's stage of development influences the effects of exposure. Most full-term infants have decreased capacity for drug metabolism until the third week of life. Drug metabolism gradually increases, so that by the 8 th to the 12 th weeks of life the rate is several times faster than in adults. It later decreases, eventually reaching the adult level of metabolism by puberty (Wisner et al, 1996).

Maximum maternal dosages that will definitely have no affect on the infant have not been established for any psychotropics. Therefore it is not possible to advise on acceptable milk/plasma ratios or safe doses. Likewise, treatment doses below which there are no clinical adverse effects or longitudinal sideeffects in the infant have also not been identified. Nevertheless, for psychotropics the arbitrary concentration in the infant's plasma of $10 \%$ of the established therapeutic maternal dose is used as the upper threshold where the risks of a particular drug's side-effects are low and treatment is accepted as safe (Bennett, 1996; Nulman et al, 2003).

If a mother on psychotropics is breast-feeding, the newborn should be systematically monitored for any possible adverse effects such as drowsiness, hypotonia, rigidity, tremor and withdrawal symptoms.

Follow-up of the infant's progress and milestones is important for the recognition of any side-effects (Box 1).

\section{Antidepressants}

Maternal distress and untreated depression adversely affect the infant and later the child (Orr \& Miller, 1995).

As far as we can ascertain, antidepressants are secreted in breast milk in very small quantities. Evidence for tricyclic antidepressants (except
Box 1 The follow-up of infants exposed to psychotropic medication

- The newborn's health should be taken into consideration when planning breast-feeding

- Preterm immature infants should not be exposed to psychotropics

- Infants' hepatic, renal and cardiac functions should be checked before they are breast-fed by mothers on psychotropic medication

- Infants older than 10 weeks are at a lower risk for adverse effects of tricyclics and there is no evidence of accumulation in the infant

- The newborn should be examined regularly for any possible adverse events of medication

- All professionals involved in the care of the infant should be informed of psychotropic medication usage

doxepin) and for selective serotonin reuptake inhibitors (SSRIs) shows that there is no clinical indication for women treated with either to stop breast-feeding, provided that the infant is healthy and its progress monitored.

Nevertheless the levels of all antidepressants in exposed infants are not well studied. The few publications are mainly case studies and small series. Research on the subject is limited and most studies do not have the necessary power to support categorical guidelines.

\section{Tricyclic antidepressants}

Studies of tricyclic antidepressants in breast-feeding have shown that drug concentration in breast milk is approximately the same as that in maternal plasma. However, the steady-state maternal plasma concentration has been accepted as a better reflection of the degree of exposure of the infant (Atkinson et $a l, 1988)$. Several studies of tricyclics have shown that neither the drugs nor their metabolites accumulated in breast-fed infants (Wisner \& Perel, 1996a). However, these were short-term studies and there has been no research on infants' long-term exposure to very low doses; this needs further investigation in longitudinal studies.

When advising a mother on breast-feeding while taking an antidepressant, a full risk/benefit analysis should be undertaken.

\section{Amitriptyline}

Several studies (e.g. Bader \& Newman, 1980; BrixenRasmussen et al, 1982) of drug levels in infant serum 
while their nursing mothers were taking therapeutic doses of amitriptyline found no detectable amitriptyline in the infants. There were no signs of adverse effects or side-effects either.

\section{Imipramine}

It has been shown that imipramine and its active metabolite desipramine produce breast-milk concentrations similar to maternal plasma levels. However, significant serum levels were not detected in the infant (Stancer \& Reed, 1986). Infants breastfed by mothers on imipramine were observed over a 2-year period and did not show any adverse effects of the drug (Misri \& Sivertz, 1991).

\section{Clomipramine}

Schimmell et al (1991) studied drug plasma levels in a breast-fed infant whose mother had taken clomipramine during pregnancy and continued after giving birth. They found that levels were high following delivery but decreased gradually and were at the lowest detectable concentration at 35 days, even though breast-feeding continued. Clomipramine has been used by breast-feeding mothers without adverse effects on the newborn.

\section{Nortriptyline}

Wisner \& Perel (1991) studied serum in breast-fed infants whose mothers were on therapeutic doses of nortriptyline and found no detectable nortriptyline or its 10-hydroxy metabolites and no adverse effects. Even when blood was taken shortly after peak maternal plasma levels there was no detectable drug or adverse event in the infants.

\section{Doxepin}

Doxepin has a longer-acting metabolite Ndesmethyldoxepin that may accumulate in infants, causing severe drowsiness and respiratory depression. There has been a near fatality in the 8-week-old baby of a mother taking $75 \mathrm{mg} /$ day (Matheson et al, 1985), but Wisner et al (1996) report no similar incidents with therapeutic doses of doxepin.

Nulman et al (2003), in their comprehensive chapter on antidepressants and breast-feeding, summarise two publications that report single cases of breast-fed infants exposed to doxepin. The concentration of doxepin and its metabolite were low in breast milk. However, one infant was sedated with depressed respiration whereas the other showed no adverse effects. Metabolic differences between infants might explain these observations.

\section{Which tricyclic?}

In view of the above, if tricyclic antidepressants need to be prescribed for breast-feeding women, amitriptyline and imipramine are the preferred choices.

\section{Trazodone}

Trazodone appears to be of lower risk because only $1 \%$ passes into the milk, although drowsiness and poor feeding have been reported. Data are limited to a few cases and caution is advised in use of the drug.

\section{Selective serotonin reuptake inhibitors}

\section{Fluoxetine}

Several authors report on infants breast-fed by mothers taking fluoxetine (Yoshida et al, 1998; Nulman et al, 2003). Fluoxetine and its active metabolite norfluoxetine were detected in all samples of maternal plasma and breast milk but their levels in infants' plasma and urine were below the lower limits of detection. All infants were developing normally and showed no abnormal findings on neurological examination (Yoshida et al, 1998). The mean combined dose of fluoxetine and norfluoxetine in the infants was below the notional $10 \%$ level of concern (Taddio et al, 1996). Maternal levels of the medication correlated highly with the fluoxetine and norfluoxetine concentration in the infants and a maternal dose of $20 \mathrm{mg} /$ day was not associated with detectable infant levels (Hale et al, 2001). However, it is important to note that inter-patient variability in these studies was quite significant.

There are also single case reports of breast-fed infants of mothers on higher doses showing sideeffects such as irritability, cyanosis, somnolence, fever, hypotonia and unresponsiveness (Lester et al, 1993; Nulman et al, 2003). But the general understanding is that although such infants may have gained less weight, they showed no clinical symptoms and did not have cognitive dysfunction when assessed with a standardised development scale (Yoshida et al, 1998; Chambers et al, 1999).

Nevertheless, close monitoring of the mother and the infant and regular examination for possible sideeffects are necessary. It is also important to alert carers and professionals involved to the possible adverse effects.

\section{Sertraline}

A relatively larger sample of infants breast-fed by mothers on sertraline has been studied. Some authors 
report that levels of the drug in the infants' serum were undetectable (Altshuler et al, 1995; Stowe et al, 1997). Others found undetectable levels in serum or levels of sertraline and its active metabolite desmethylsertraline of less than $10 \mathrm{ng} / \mathrm{ml}$ (Altshuler et al, 2001). Epperson et al (2001) studied serotonin (5-HT) transport in nine pairs of mothers and infants and concluded that at clinical doses of sertraline, platelet 5-HT uptake in nursing infants was unaltered and mothers taking sertraline could breast-feed without appreciably affecting peripheral or central 5-HT transport in their infants.

In view of the larger samples, undetectable serum levels of sertraline and cumulative research evidence indicating no significant adverse effects on the infant, this medication has been recommended in the USA as the first-line treatment for breast-feeding mothers (Altshuler et al, 2001).

\section{Paroxetine}

The concentration of paroxetine in breast milk is similar to that in the mother's plasma (Kaye et al, 1989). Less than $1 \%$ of the steady state concentration in maternal plasma is transferred to the breast-fed infant, and this amount causes no adverse effects (Duncan \& Taylor, 1995). Evidence shows that paroxetine has a lower milk/plasma ratio than fluoxetine and sertraline (Misri et al, 2000). Merlob et al (2004) studied 27 mothers taking paroxetine while breast-feeding, comparing them with a control group. They found no notable adverse events and the infants reached milestones at similar ages. They advised the lowest possible single bed time dose for the mothers and regular clinical follow-up of the infants.

\section{Citalopram}

Citalopram concentration in breast milk has been studied in smaller samples and the milk/plasma

\section{Box 2 Antidepressants and breast-feeding}

- For women taking antidepressants the treatment strategy while breast-feeding has been clearly established

- Tricyclics such as amitryptyline, imipramine, nortriptyline and clomipramine are safe during breast-feeding

- SSRIs such as fluoxetine, sertraline, paroxetine and citalopram are safe during breastfeeding

- It is advisable to discontinue MAOIs, replacing them with a newer drug if necessary ratio has been found to be relatively high, as is the calculated infant dose (Jensen et al, 1997; Spigset et al, 1997; Schmidt et al, 2000). None of the infants studied displayed detectable levels of citalopram or its metabolite, but possible symptoms such as somnolence, restlessness and irritability have been attributed to maternal citalopram intake. However, Lee et al (2004a), in their prospective observational cohort, studied the frequency of adverse events in 31 infants exposed to maternal citalopram through breast-feeding. Citalopram was found to be safe and there were no statistically significant adverse events or any developmental problems.

At the time of publication, there were no reports on escitalopram use during breast-feeding.

\section{Venlafaxine}

Ilett et al (1998) have studied the levels of venlafaxine and its metabolites in breast milk and in exposed infants at different times during the first 8 months of life. Although venlafaxine accumulated in breast milk, infant plasma levels were only $3.2 \%$. This is clearly below the notional $10 \%$ level of concern and therefore was accepted as undetectable. There were no adverse events or side-effects in any of the infants.

\section{Mirtazapine, nefazodone and bupropion}

There are no studies of mirtazapine, nefazodone or bupropion use during breast-feeding. Nevertheless, all three antidepressants bind to serum protein. All three have metabolites and therefore if used by breast-feeding mothers, infants should be monitored for potential side-effects (Heath \& Yonkers, 2001).

\section{Monoamine oxidase inhibitors}

The side-effects of monoamine oxidase inhibitors (MAOIs) in adults are well documented, but no studies of their side-effects in infants have been published. Likewise, no cases of risk or damage caused to infants by MAOIs have been reported. Regardless of this lack of evidence of side-effects in infants, now that several new antidepressants are on offer psychiatrists should review their treatment plans and discontinue MAOIs in mothers planning to breast-feed.

Box 2 summarises key points regarding antidepressants and breast-feeding.

\section{Conventional antipsychotics}

In their comprehensive reviews, Yoshida et al (1999) and Burt et al (2001) have summarised the findings of the few reported cases involving breast-fed infants and conventional antipsychotics (chlorpromazine, 
trifluoperazine or perphenazine) and atypicals (clozapine or olanzapine). Only a few of those infants showed side-effects, such as drowsiness, lethargy and possible gastrointestinal complaints.

Yoshida et al (1997) studied 12 mothers who breast-fed while on haloperidol, chlorpromazine or trifluoperazine postnatally. It is important to note that none had received antipsychotics during pregnancy. The amount of the drug in their plasma correlated with the amount in breast milk. Very small amounts were detected in their infants' plasma and urine but no toxic effects were noted. High-potency antipsychotics have been recommended during breast-feeding because they cause less sedation and less autonomic effect (Wisner \& Perel, 1996b).

In a haloperidol study, Whalley et al (1981) found that the concentration of the drug in the breast milk of nursing women was about two-thirds of that in their serum. Their infants showed no side-effects of the medication and no behavioural teratology.

\section{Atypical antipsychotics Olanzapine}

Mothers breast-feeding while on olanzapine and their infants have been studied by Gardiner et al (2003). They concluded that infants exposed to olanzapine showed plasma levels much below the $10 \%$ level of concern. Mean infant exposure (mg/ $\mathrm{kg}$ ) at steady state was estimated to be $1.8 \%$ of the maternal olanzapine dosage ( $\mathrm{mg} / \mathrm{kg}$ ). In the infants' plasma, olanzapine levels were below the detection limit and the babies showed no adverse effects. Although these limited data suggest that olanzapine is safe during breast-feeding, mothers are advised not to breast-feed while taking it and all decisions need to be made after detailed individual risk/benefit analysis.

\section{Risperidone}

Ilett et al (2004) studied the plasma levels of an infant breast-fed by a mother taking risperidone and showed that the relative infant dose was below the notional $10 \%$ level of concern. They concluded that maternal risperidone therapy is unlikely to be a significant hazard for breast-fed infants in the short term. However, the decision whether a woman may breast-feed should be made after individual risk/ benefit analysis.

\section{Quetiapine}

Possible adverse effects of quetiapine have not been studied in breast-fed infants. Post-marketing pharmacovigilance studies include no cases of a mother breast-feeding while on quetiapine. Safety considerations dictate that women taking the drug should be advised not to breast-feed because of limited experience with the agent. However, in a case reported by Lee et al (2004b) the level of infant exposure to quetiapine in breast milk appeared to be too small for significant pharmacological effects.

\section{Clozapine}

There are several reported cases of clozapine use during pregnancy and breast-feeding (Barnas et al, 1994; Dev \& Krupp, 1995). Clozapine was clearly found to accumulate in breast milk and foetal serum. The higher concentration in foetal serum was explained by the higher concentration of albumin in foetal blood.

Dev \& Krupp (1995) reported on four infants breast-fed by mothers receiving clozapine: two of the babies had no apparent adverse effects, one developed agranulocytosis but recovered spontaneously when breast-feeding was discontinued and the fourth became excessively sleepy.

Because of its obvious accumulation in breast milk, clozapine is one of the few psychotropics contraindicated during breast-feeding.

\section{Amisulpride}

There are no studies to demonstrate whether amisulpride is excreted in breast milk and whether it causes adverse events in the infant. Women should therefore be advised not to breast-feed while on amisulpride.

Information regarding breast-feeding while on antipsychotic medication is summarised in Box 3.

\section{Box 3 Antipsychotics and breast-feeding}

- Conventional antipsychotics have been used for decades and the accumulated data show that they are safe during breast-feeding

- New information is starting to emerge about some atypical antipsychotics such as olanzapine and risperidone but their safety has yet to be established

- There is currently no information on quetiapine and amisulpride and therefore it is not safe to expose newborns to these medications

- Clozapine accumulates in breast milk and is contraindicated during breast-feeding 


\section{Antimanic drugs \\ Lithium}

Lithium salts are excreted into the breast milk at a level of $40-50 \%$ of maternal serum levels (Sykes et al, 1976). Infant serum levels are equal to breast milk levels and at times can rise to $200 \%$ of the maternal serum concentration. It is well documented that infants can develop several signs and symptoms of toxicity, including cyanosis and hypothermia, if breast-fed by mothers on lithium treatment (Chaudron \& Jefferson 2000). Therefore lithium is contraindicated during breast-feeding.

\section{Carbamazepine}

Carbamazepine and its active metabolite are found in breast milk in significant quantities and the medication is generally detected in infant plasma. Infant serum carbamazepine levels range from 10 to $60 \%$ of maternal serum levels. Nevertheless, there is little evidence of adverse events in infants and little evidence for discontinuation of the drug by mothers taking it as an anti-epileptic (Yoshida et $a l, 1999)$ However, transient hepatic toxicity such as hyperbilirubinaemia and high concentrations of gamma-glutamyl-transferase have been reported in neonates exposed to carbamazepine during breast-feeding (Merlob et al, 1992). Caution should be exercised and a risk/benefit analysis carried out before mothers are allowed to breast-feed while on carbamazepine.

\section{Sodium valproate}

The concentration of valproate in the mother's milk is 3\% or less than the maternal plasma levels (Nau et al, 1981). Neurologists who prescribe valproate for its anti-epileptic role advise mental health professionals to complete a detailed risk/benefit analysis before allowing the mother to breast-feed the infant. There are no reports of major adverse events in children breast-fed by mothers taking sodium valproate (Chaudron \& Jefferson, 2000; Piontek et al, 2000).

\section{Hypnotics and anxiolytics \\ Benzodiazepines}

Benzodiazepines are addictive drugs and therefore should not be prescribed as anxiolytics or sedatives on a long-term basis. Women should not be started on benzodiazepines if they are breast-feeding. Ideally, women should be encouraged to stop taking these drugs before they become pregnant.
Benzodiazepines taken by the mother during pregnancy should be withdrawn with caution, as infants who have been under the influence of these drugs in utero may have acquired drug dependence and suffer withdrawal symptoms if the drugs are no longer available to them.

Benzodiazepines are excreted in breast milk at a low milk/plasma ratio (Yoshida et al, 1999). Cases of infants breast-fed by mothers on clonazepam or temazepam have shown no measurable drug levels in the infants' serum and no adverse effects were reported. Birnbaum et al (1999) analysed levels of psychotropics, including benzodiazepines, in breast-fed infants. In infants whose mothers were not on medication during pregnancy but started only after delivery, serum concentrations were below the laboratory limit of detection. Only in infants who were exposed to benzodiazepines during pregnancy could the medication be detected in their serum. These data support the low incidence of infant toxicity and adverse effects associated with benzodiazepine use during breastfeeding.

Nevertheless, repeated doses of long-acting benzodiazepines can produce lethargy, poor suckling and weight loss, and infants need to be monitored.

\section{Others}

Buspirone, zaleplon and zopiclone should all be avoided because they are excreted in breast milk (Bazire, 2003). Zopiclone in particular is contraindicated during breast-feeding as it is excreted in the milk at up to $50 \%$ of maternal plasma levels (Matheson et al, 1990), although a single dose may not cause accumulation.

Zolpidem is accepted as compatible with breastfeeding because of its low lipophilic properties and rapid excretion (Bazire, 2003).

Box 4 summarises the position for antimanics, hypnotics and anxiolytics in breast-feeding.

\section{Risk/benefit analysis}

Mental health professionals involved in the management of women's psychiatric problems during the perinatal period have to make an individualised risk/benefit analysis to advise each woman on medication while pregnant (Kohen, 2004) and the possibility of safe breast-feeding.

The first step in the analysis is to record details of the psychiatric condition, the severity and frequency of the problems, the level of family support, and the woman's general cooperation with treatment (previous attendance for appointments 
Box 4 Antimanics, hypnotics and anxiolytics during breast-feeding

- Lithium is contraindicated during breastfeeding

- There is little evidence of adverse events in infants breast-fed by mothers taking carbamazepine or sodium valproate, although transient hepatic toxicity is possible with the former

- It is unsafe to expose infants to repeated doses of long-acting benzodiazepines through breast-milk

- Buspirone, zaleplon and zopiclone are contraindicated during breast-feeding

- Zolpidem is safe during breast-feeding

and adherence to medication regimens). A number of other factors are important: the reliability of informants, the level of understanding of early warning signs, good rapport with care coordinators and health visitors, and involvement of statutory and voluntary organisations. The amount of medication needed during the pregnancy and the need for monotherapy will play a role in the decision.

The physical health and maturity of the infant are clearly important. It is also essential that the mother should be well enough to look after her newborn and not go through a crisis and need hospitalisation. It may be more important to give the mother the amount and number of psychotropics necessary to keep her well in the community with her baby than to allow her to remain undertreated while breast-feeding. A healthier mother will be able to care for her bottle-fed newborn but an unwell mother will neglect her breast-fed infant.

\section{Conclusions}

It is quite clear from this overview that, given the limited data regarding breast-milk levels and serum concentrations of psychotropic medication in breastfeeding infants, it is difficult to reach a firm conclusion on the safety of breast-feeding (Box 5). And with the exception of clozapine and lithium salts, which are clearly contraindicated, no single agent seems to be of greater risk than others.

It is also well established that none of the drugs discussed above is established as being completely free of side-effects. Therefore all drugs should be viewed with caution before breast-feeding. Still, there are documented differences between drugs within the same class and there is no class action in relation to breast-feeding.

All psychotropics are secreted into the breast milk but concentrations and effects can vary. The results of earlier clinical reports and case studies and the more recent studies with larger patient samples lead to the tentative conclusion that, for most psychotropics, low doses of a single drug are relatively safe during breast-feeding.

The decision to breast-feed must be made jointly by the mother, the family, the psychiatrist and the health visitor involved in the mother's psychiatric management. The benefits of breast-feeding for the mother and the infant should outweigh the risks of exposing the infant to small doses of the medication. Mothers who need to be on two or more psychotropics simultaneously or who have to take medication at the upper end of the recommended dose range should be advised not to breast-feed. Women on atypical antipsychotics are also advised not to breast-feed because of the paucity of experience with these medications. It is essential that infants are monitored by health visitors and paediatricians for any possible adverse events.

Breast-feeding while taking psychotropic medication is an important issue that has not received the attention it deserves. It is clear that there is very limited research and data on the subject and most of the information comes from a small number of studies based on a few cases or case reports. With the revived interest in perinatal psychiatry, I hope that there will be a revival of the subject and controlled and systematic research will be able to address some of the questions yet to be resolved.

\section{Box 5 Key points}

- The decision to prescribe antipsychotics to breast-feeding women should depend on individual risk/benefit analysis

- The current available research does not allow any absolute and clear recommendation because much of the work on psychotropic medication in breast-feeding is limited to single case reports, small series and naturalistic data collection

- Causes and consequences of different adverse events are not yet widely studied

- Non-psychiatric professionals, especially health visitors, need to be trained to recognise adverse events

- There is a need for further research and accumulation of experience 


\section{References}

Altshuler, L. L. Burt, V. K., McMullen, M., et al (1995) Breast feeding and sertraline: a 24 hour analysis. Journal of Clinical Psychiatry, 56, 243-245.

Altshuler, L. L., Cohen, L. S., Moline, M. L., et al (2001) The Expert Consensus Guidelines Series: Treatment of Depression in Women. Postgraduate Medicine Special Report. New York: McGraw Hill. http://www.psychguides.com/ecgs6.php

Atkinson, H. C., Begg, E. J. \& Darlow, B. A. (1988) Drugs in human milk: clinical pharmacokinetic considerations. Clinical Pharmacokinetics, 14, 217-240.

Bader, T. F. \& Newman, K. (1980) Amitriptyline in human breast milk and nursing infant's serum. American Journal of Psychiatry, 137, 855-856.

Barnas, C., Bergant, A., Hummer, M., et al (1994) Clozapine concentration in maternal plasma, amniotic fluid and breast milk (letter). American Journal of Psychiatry, 151, 945.

Bazire, S. (2003) Psychotropic Drug Directory. Salisbury: Fivepin Publishing.

Bennett, P. N. (1996) Use of the monographs on drugs. In Drugs and Human Lactation (ed. P. N. Bennett), pp. 67-74. Amsterdam: Elsevier

Birnbaum, C. S., Cohen, L. S., Bailey, J. W., et al (1999) Serum concentration of antidepressants and benzodiazepines in nursing infants: a case series. Pediatrics, 104, e11.

Brixen-Rasmussen, L., Halgrener, J. \& Jorgensen, A. (1982) Amitriptyline and nortriptyline excretion in human breast milk. Psychopharmacology (Berlin), 76, 94-95.

Burt, V. K., Suri, R., Altshuler, L., et al (2001) The use of psychotropic medications during breast-feeding. American Journal of Psychiatry, 158, 1001-1009.

Chambers, C. D., Anderson, P. O., Thomas, R. G., et al (1999) Weight gain in infants breast-fed by mothers who take fluoxetine. Paediatrics, 104. 61.

Chaudron, L. H. \& Jefferson, J. W. (2000) Mood stabilizers during breast-feeding: a review. Journal of Clinical Psychiatry, 61, 79-90.

Dev, V. \& Krupp, P. (1995) Adverse event profile and safety of clozapine. Reviews in Contemporary Pharmacotherapy, $\mathbf{6}$ 197-208.

Duncan, D. \& Taylor, D. (1995) Which antidepressants are safe to use in breast-feeding mothers? Psychiatric Bulletin $19,551-552$

Epperson, N., Czarkowski, K. A., Ward-O'Brien, D., et al (2001) Maternal sertraline treatment and serotonin transport in breast-feeding mother-infant pairs. American Journal of Psychiatry, 158, 1631-1637.

Fergusson, D. M., Beautrais, A. L. \& Silva, P. A. (1982) Breastfeeding and cognitive development in the first seven years of life. Social Science and Medicine, 16, 1705-1708.

Gardiner, S. J., Kristensen, J. H., Begg, E. J., et al (2003) Transfer of olanzapine into breast milk, calculation of infant drug dose, and effect on breast-fed infants. American Journal of Psychiatry, 160, 1428-1431.

Hale, T.W., Shum, S. \& Grossberg, M. (2001) Fluoxetine toxicity in a breast-fed infant. Clinical Pediatrics (Philadelphia), 40, 681-684

Heath, A. C. \& Yonkers, K. A. (2001) Somatic treatments in depression: concerns during pregnancy and breastfeeding. In Management of Psychiatric Disorders in Pregnancy (eds K. Yonkers \& B. Little), pp. 82-104. London: Hodder Arnold.

Ilett, K. F., Hackett, L. P., Dusci, L. J., et al (1998) Distribution and excretion of venlafaxine and O-desmethylvenlafaxine in human milk. British Journal of Clinical Pharmacology, 45, $459-462$

Ilett, K. F., Hackett, L. P., Kristensen, J. H., et al (2004) Transfer of risperidone and 9-hydroxyrisperidone into human milk. Annals of Pharmacotherapy, 38, 273-276.

Jensen, P. N., Olesen, O. V., Bertelsen, A., et al (1997) Citalopram and desmethylcitalopram concentration in breastmilk and serum of mother and infant. Therapeutic Drug Monitoring, 19, 236-239.
Kaye, C. M., Haddock, R. E., Langley, P. F., et al (1989) A review of the metabolism and pharmacokinetics of paroxetine in man. Acta Psychiatrica Scandinavica Supplementum, 350, 60-75.

Kohen, D. (2004) Psychotropic medication in pregnancy. Advances in Psychiatric Treatment, 10, 59-66.

Lee, A., Woo, J. \& Ito, S. (2004a) Frequency of infant adverse events that are associated with citalopram use during breast-feeding. American Journal of Obstetrics and Gynecology, 190, 218-221.

Lee, A., Giesbrecht, E., Dunn, E., et al (2004b) Excretion of quetiapine in breast milk. American Journal of Psychiatry, 161, 1715-1716.

Lester, B. M., Cucca, J., Lynne, A., et al (1993) Possible association between fluoxetine hydrochloride and colic in an infant. Journal of the American Academy of Child and Adolescent Psychiatry, 32, 1253-1255.

Lucas, A., Morley, R., Cole, T. J., et al (1992) Breast feeding and subsequent intelligence quotient in children born preterm. Lancet, 339, 261-264.

Matheson, I., Panda, H. \& Alertsen, A. R. (1985) Respiratory depression caused by $\mathrm{N}$-desmethyldoxepin in breast milk (letter). Lancet, 2, 1124.

Matheson, I., Sande, H. A. \& Gaillot, J. (1990) The excretion of zopiclone into breast milk. British Journal of Clinical Pharmacology, 30, 267-271.

Merlob, P., Mor, M. \& Litwin, A. (1992) Transient hepatic dysfunction in an infant of an epileptic mother treated with carbamazepine during pregnancy and breast-feeding. Annals of Pharmacotherapy, 26, 1563-1565.

Merlob, P., Stahl, B. \& Sulkes, J. (2004) Paroxetine during breast-feeding: infant weight gain and maternal adherence to counsel. European Journal of Pediatrics, 163, 135-139.

Misri, S. \& Sivertz, K. (1991) Tricyclic drugs in pregnancy and lactation: a preliminary report. International Journal of Psychiatry in Medicine, 21, 157-171.

Misri, S., Kim, J., Riggs, K. W., et al (2000) Paroxetine levels in postpartum depressed women, breast milk and infant serum. Journal of Clinical Psychiatry, 61, 828-832.

Nau, H., Rating, D., Koch, S., et al (1981) Valproic acid and its metabolite: placental transfer, neonatal pharmacokinetics, transfer via mother's milk and clinical status in neonates of epileptic mothers. Journal of Pharmacology and Experimental Therapeutics, 219, 768-777.

Nulman, I., Ito, S. \& Koren, G. (2003) Antidepressant medication and breast-feeding. In Handbook of Female Psychophamacology (eds M. Steiner \& G. Koren), pp. 103115. London: Martin Dunitz.

Orr, S. T. \& Miller, C. A. (1995) Maternal depressive symptoms and the risk of poor pregnancy outcome. Epidemiologic Reviews, 17, 165-171.

Piontek, C., Baab, S., Peindel, K. S., et al (2000) Serum valproate levels in 6 breast-feeding mother-infant pairs. Journal of Clinical Psychiatry, 61, 170-172.

Schimmell, M. S., Katz, E. Z., Shaag, Y., et al (1991) Toxic neonatal effects following maternal clomipramine therapy. Journal of Toxicology: Clinical Toxicology, 29, 479-484.

Schmidt, K., Olesen, O. V. \& Jensenn, P. N. (2000) Citalopram and breast-feeding: serum concentration and side-effects in the infant. Biological Psychiatry, 47, 164-165.

Spigset, O., Carleborg, L., Ohman, R., et al (1997) Excretion of citalopram in breast milk. British Journal of Clinical Pharmacology, 44, 295-298.

Stancer, H. C. \& Reed, K. L. (1986) Desipramine and 2hydroxydesipramine in human breast milk and the nursing infant's serum. American Journal of Psychiatry, 143, 15971600.

Stowe, Z. N., Owens, M. J., Landry, J. C., et al (1997) Sertraline and desmethylsertraline in human breast milk and nursing infants. American Journal of Psychiatry, 154, 1255-1260.

Suri, R. A., Altshuler, L. L., Burt, V. K., et al (1998) Managing psychiatric medications in the breast-feeding woman. Medscape Women's Health, 3, 1-10.

Sykes, P. A., Quarrie, J. \& Alexander, F. W. (1976) Lithium carbonate and breast-feeding. BMJ, 2, 1299. 
Taddio, A., Ito, S. \& Koren, G. (1996) Excretion of fluoxetine and its metabolite, norfluoxetine in human breastmilk. Journal of Clinical Pharmacology, 36, 42-47.

Whalley, L. J., Blain, P. G. \& Prime, J. K. (1981) Haloperidol secreted in breast milk. BMI, 282, 1746-1747.

Wisner, K. L. \& Perel, J. M. (1991) Serum nortriptyline levels in nursing mothers and their infants. American Journal of Psychiatry, 148, 1234-1236.

Wisner, K. L. \& Perel, J. M. (1996a) Nortriptyline treatment of breast-feeding women (letter). American Journal of Psychiatry, 153, 295.

Wisner, K. L. \& Perel, J. M. (1996b) Psychopharmacological treatment during pregnancy and lactation. In Psychopharmacology and Women: Sex, Gender and Hormones (eds M. Jensvold, U. Halbreich \& J. Hamilton), pp. 191-224. Washington, DC: American Psychiatric Publishing.

Wisner, K. L., Perel, J. M. \& Findling, R. L. (1996) Antidepressant treatment during breast feeding. American Journal of Psychiatry, 153, 1132-1137.

Yoshida, K., Smith, B., Craggs, M., et al (1997) Neuroleptic drugs in breast milk: a study of pharmacokinetics and of possible adverse effects in breast-fed infants. Psychological Medicine, 28, 81-91.

Yoshida, K., Smith, B., Craggs, M., et al (1998) Fluoxetine in breast-milk and development outcome of breast-fed infants. British Journal of Psychiatry, 172, 175-178.

Yoshida, K., Smith, B. \& Kumar, R. C. (1999) Psychotropic drugs in mothers' milk: a comprehensive review of assay methods, pharmacokinetics and safety of breast-feeding. Journal of Psychopharmacology, 13, 64-80.

\section{MCQs}

1 In treating mothers with mental health problems:

a decisions concerning the use of psychotropic medication in pregnancy are complicated

b decisions regarding the initiation of pharmacotherapy require consideration of its effects on the newborn

c the evidence regarding safety of breast-feeding while taking medication is well established

d psychiatrists should be able to discuss with their patients the risks and benefits of taking psychotropics while breast-feeding

e the dose below where there is no clinical effect in infants is known for most drugs.

2 It is well esatblished that:

a there are no known side-effects in breast-feeding infants exposed to imipramine, desipramine or nortriptyline b amitriptyline has well-known adverse effects in exposed infants

c infants exposed to clomipramine show no side-effects

d dothiepin has been investigated for its long-term side-effects in infants and has been shown to be safe

e respiratory depression can be one of the reversible side-effects following exposure to tricyclic antidepressants.

3 The following facts are correct:

a fluoxetine, sertraline and citalopram levels have been investigated in infants exposed through breastfeeding

$\mathrm{b}$ it is reassuring to see that with some SSRIs serum levels in infants are not detectable

c exposure of infants to SSRIs through breast-feeding can cause developmental delay

$\mathrm{d}$ there is an increased frequency of adverse events such as irritability and restlessness in infants exposed to SSRIs

e there are data to support liver and kidney dysfunction in infants breast-fed by mothers on SSRIs.

4 Regarding mothers who are breast-feeding:

a benzodiazepines are not excreted in breast milk

$\mathrm{b}$ benzodiazepines can produce lethargy and weight loss in the infant

c short-acting benzodiazepines should be preferred to long-acting ones

d prescription of benzodiazepines jointly with SSRIs would be a safer approach

e benzodiazepines with doxepin is an advisable treatment.

5 In the management of breast-feeding women:

a sedating drugs with long half-lives should be avoided in breast-feeding mothers

b drugs should be avoided if the infant is premature or has hepatic, renal or cardiac failure

c avoiding interacting drugs that raise plasma levels is important

d drug effects on the development of the infant are well documented in several recent papers

e several formal studies give good indication of infant plasma levels of the commonly used psychotropics. 